\title{
Los procesos de reproducción cultural en Mesoamérica, una perspectiva transdisciplinaria
}

\author{
Horacio E. Mendizábal García
}

\section{Resumen}

El artículo aborda una problematización teórico-metodológica desde el pensamiento transdisciplinario sobre los procesos de reproducción cultural. Se utilizan dichos planteamientos para la discusión sobre el concepto Mesoamérica y su validez teórico-metodológica para las ciencias sociales.

Palabras clave: mesoamérica, complejidad, transdisciplina.

\section{Cultural repruduction processes in Mesoamerica,}

\section{A TRANSDISCIPLINAR PERSPECTIVE}

\begin{abstract}
The article addresses a theoretical-methodological problematization about the processes of cultural reproduction seen from the optics of transdisciplinar thinking. These approaches are used for the discussion of the Mesoamerica concept and its theoretical-methodological validity for the social sciences.
\end{abstract}

Key words: mesoamerican, complexity, transdiscipline.

\section{Horacio E. Mendizábal García}

alomorfo@yahoo.com.mx

Licenciado en Lingüista por parte de la Escuela Nacional de Antropología e Historia (ENAH). Actualmente cursa la maestría en Antropología Social en la línea de investigación sobre Análisis del discurso y Semiótica de la cultura en la ENAH. Sus temas de investigación son el análisis de los modos de reproducción subjetiva plasmados en las prácticas semiótico-discursivas humanas, observados desde una articulación entre la epistemología de la complejidad y la semiótica de la cultura. Ha participado en investigaciones sobre rituales mayas y modelos educativos mesoamericanos, con relación a las lógicas simbólico-semióticas de reproducción subjetiva. 


\section{Mesoamérica una conceptualización en conflicto}

El presente artículo aborda una discusión sobre los procesos de reproducción cultural desde la perspectiva transdiciplinar y del pensamiento complejo. Para tal fin es necesario abordar la relación entre las prácticas de los seres humanos y el conocimiento histórico articulado a ellas. Conocimiento que se codifica en muchos lenguajes y materialidades parar guardar la información necesaria que da sentido a lo que hacemos.

Como es de suponerse, los procesos de reproducción cultural existen relacionados a un contexto particular que es parte inseparable de su desarrollo. Por lo tanto, para ejemplificar la discusión teórica se recurre al concepto Mesoamérica, como el contexto simbólico y práctico alrededor del cual girarán nuestras reflexiones sobre la reproducción de la cultura.

Mesoamérica es una construcción teórica que refiere a un área geográfica que abarca desde el centro mexicano hasta las tierras costarricenses. En un inicio, el concepto se basaba principalmente en la evidencia material como son los distintos sitios arqueológicos que presentaban características compartidas. Existen otros tipos de evidencias utilizadas para sustentar el concepto mesoamericano, como son las distintas familias lingüísticas que todavía se hablan en dichos territorios. Desde los tiempos prehispánicos existía una amplia diversidad que puede observarse todavía en la pluralidad lingüística.

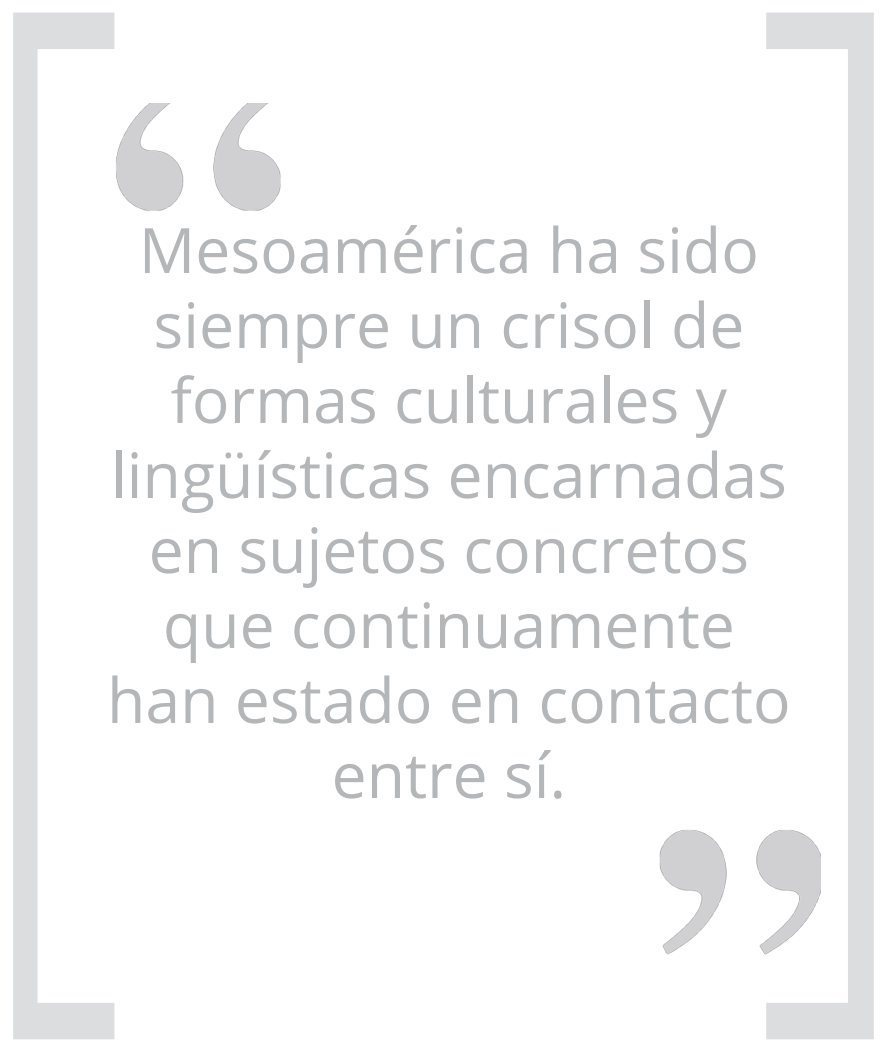

Mesoamérica se puede entender actualmente desde diversas perspectivas. El término fue acuñado por Paul Kirchhoff en 1943 como un "intento de señalar lo que tenían en común los pueblos y las culturas de una determinada parte del continente americano, y lo que los separaba de los demás" (2000: 15). Actualmente existe una discusión sobre la validez del concepto, ya que la diversidad de grupos étnicos es tan grande que ha llevado a algunos investigadores (como es el caso de Jauregui, 2008) a poner en duda la existencia de un vínculo cultural a nivel regional.

Sin embargo, el pensamiento complejo y la transdisciplina permiten pensar Mesoamérica de otra forma, es decir, comprender la unitas multiplex que implica entender en un continuum recursivo la unidad <-> diversidad. La relación entre la historia contemporánea y las formas culturales de largo tiempo -entendidas como ese entramado complejo que vincula la vida y práctica de los sujetos en el territorio-, es lo que hace posible el concepto de Mesoamérica como una matriz macro (un conjunto articulado) de relaciones simbólicas que codifican tanto al territorio, 

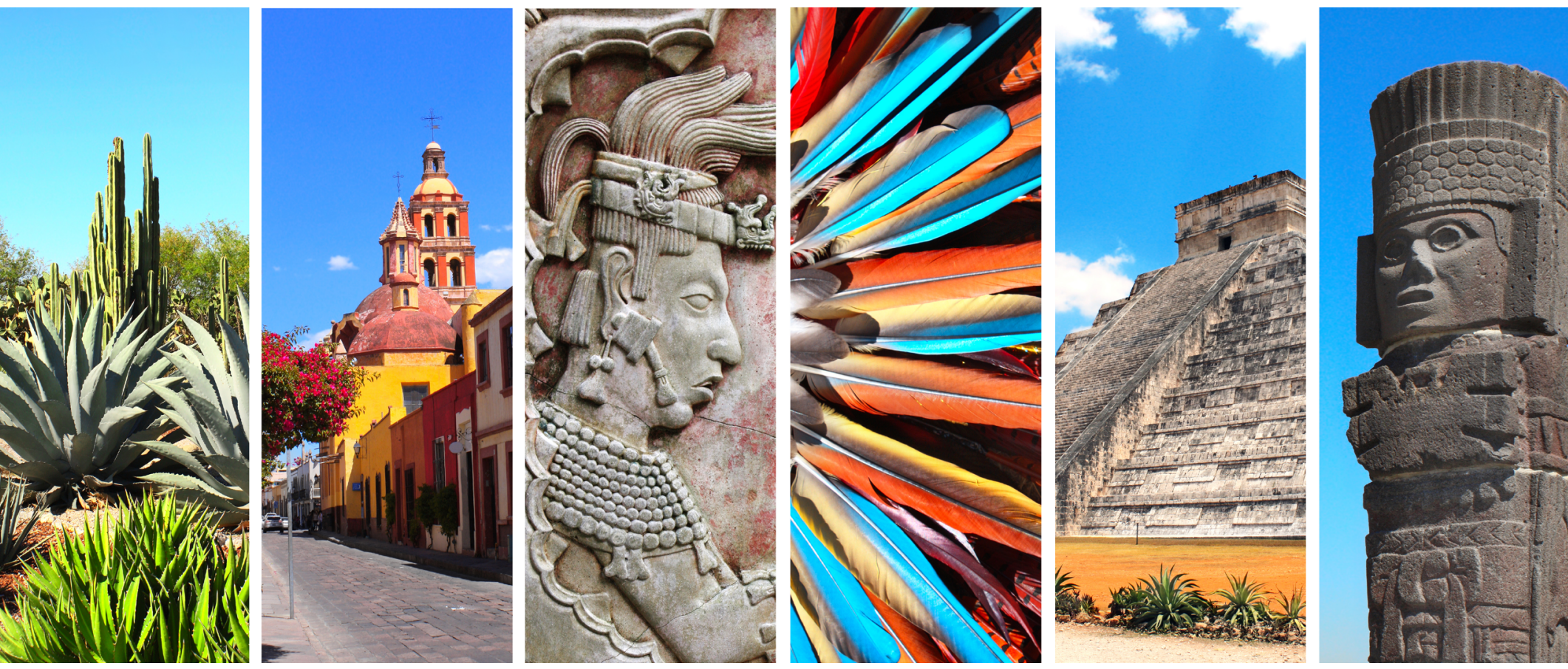

\section{La reproducción cultural desde una perspectiva transdisciplinaria}

Podemos definir brevemente la reproducción cultural como aquellas prácticas en que los sujetos recrean las historias con las cuales explican la manera en que viven, cómo entienden el mundo y qué lugar sienten tener en él. Historias ligadas tanto al presente, como al pasado de sus vidas y, por lo tanto, a su futuro.

Como puede apreciarse, estudiar los procesos de reproducción cultural involucra muchas dimensiones de la vida de los sujetos complejos. Dimensiones que van desde la manera en que alguien consigue lo necesario para seguir viviendo, como el agua o la comida, hasta el conocimiento sobre quienes sentimos ser. Para enfrentar tal cantidad de factores que atraviesan la reproducción 
cultural, es muy útil apoyarse en los planteamientos del pensamiento complejo y la transdisciplinariedad.

En este sentido, podemos entender lo transdisciplinario como una manera de construir conceptos y categorías para pensar problemas de estudio de manera cruzada y entretejida en muchas lógicas y dimensiones analíticas, como es el caso de la reproducción cultural. Sin embargo, ¿cómo pueden observarse estos procesos que unen las ideas del pasado, el presente y el futuro en la vida de los seres humanos? Para resolver tal interrogante recurrimos a otra categoría construida por Haidar desde el pensamiento complejo: la práctica semióticodiscursiva (Haidar, 2006: 75-76).

Siguiendo este razonamiento, entendemos como práctica semiótico-discursiva a las acciones humanas, las cuales están siempre entretejidas en muchas lógicas que generan su sentido-significado. Actos humanos, con dimensiones conscientes e inconscientes, que son en sí mismos expresiones de conocimiento y memoria de los grupos humanos que forman a las personas. De esta manera, puede decirse que existe una relación entre cultura, conocimiento y memoria que es parte de los procesos mediante los cuales los sujetos humanos se reproducen en el mundo.

Para acercarnos al vínculo entre conocimiento y cultura expresado en las prácticas subjetivas, recurrimos al pensamiento complejo y a la transdisciplinariedad, como se explicita en la siguiente cita de Morín:

Las aptitudes cognitivas humanas solo pueden desarrollarse en el seno de una cultura que ha producido, conservado, transmitido un lenguaje, una lógica, un capital de saberes, de criterios de verdad. Es en este marco donde el espíritu humano elabora y organiza su conocimiento utilizando los medios culturales de que dispone [...] la actividad cognitiva se ha visto en interacciones a la vez complementarias y antagonistas con la ética, el mito, la religión, la política, y el poder con frecuencia ha controlado al saber para controlar el poder del saber (Morín, 2006: 20).

Como podemos observar, los procesos de reproducción tanto cultural como del conocimiento están siempre ligados a lógicas particulares mediante las cuales se expresan. Lógicas de reproducción cultural que abarcan los significados de las prácticas de los sujetos.

Es esclarecedor regresar al ejemplo de Mesoamérica para entender la reproducción cultural desde los planteamientos de la transdisciplina y el pensamiento complejo. Tomando en cuenta la grandísima diversidad cultural, la cual es parte del argumento de Jauregui para negar el concepto de Mesoamérica, el punto de vista generado por la transdisciplina permite abrir nuevas perspectivas para pensar Mesoamérica.

Hay múltiples evidencias que prueban la existencia de una dimensión semiótico-simbólica compartida a lo largo del área que abordamos. Es decir, las lógicas que dan significado a las acciones de las personas, las cuales se relacionan 
a la historia compartida por los sujetos que habitan Mesoamérica, pueden ser observadas en la vida cotidiana. Un ejemplo de lo que afirmamos, es la cantidad de especies de maíz que existen en el territorio. El maíz es una planta domesticada desde hace miles de años en la región mesoamericana. Dicho proceso de domesticación implica sistemas de reproducción y adaptación del conocimiento y la cultura heredados a lo largo de generaciones. En Guatemala es posible encontrar variedades de maíz adaptadas a los distintos climas del país: existe maíz que se puede cosechar tres veces al año, el cual se cultiva en áreas donde la humedad permite tal proceso; hay también maíz de montaña, el cual crece en el altiplano y es más resistente al frío. Pueden encontrarse distintas variedades de color que van del blanco al azul oscuro, pasando por el rojo y el amarillo. La variedad no es solo biológica, también los modos en que se prepara en las cocinas son muchos.

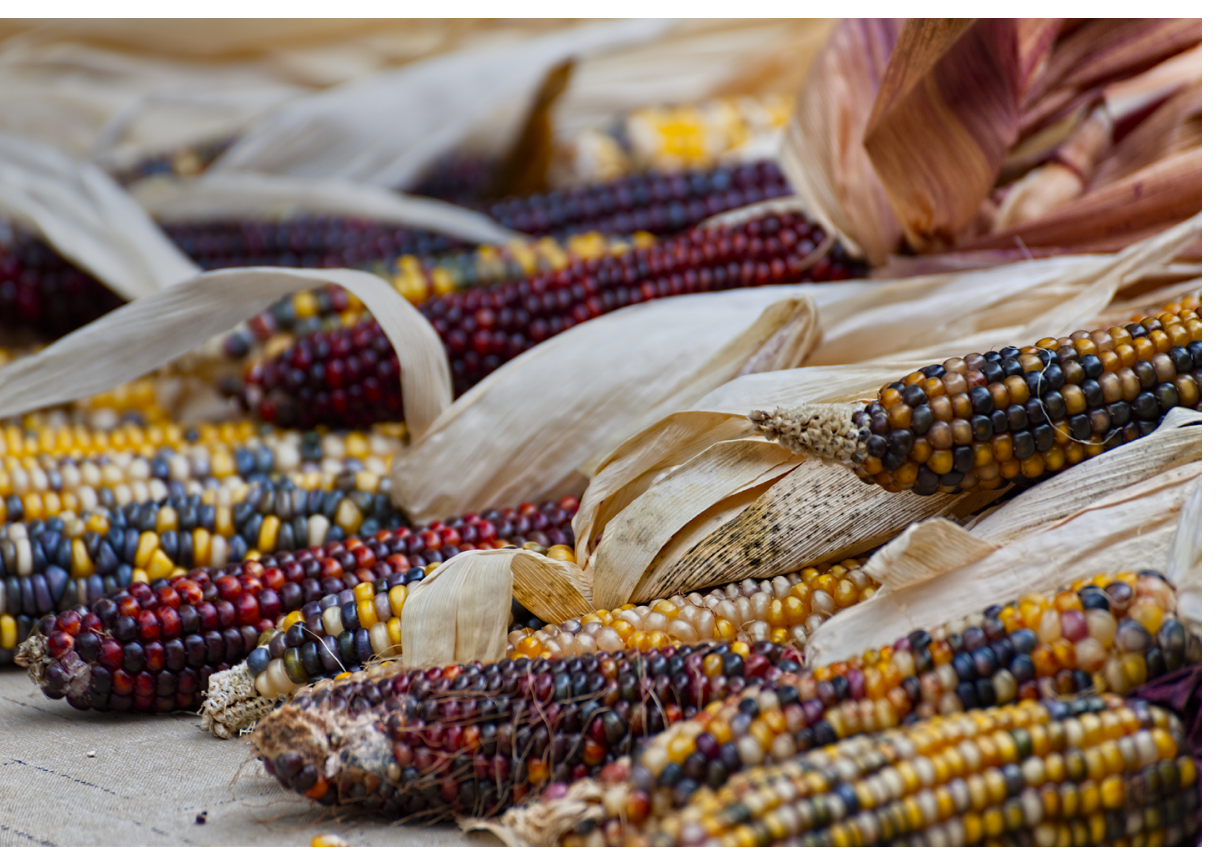

Lo anterior es observable no solo en Guatemala, ya que el estado de Tlaxcala en México lleva en las bases léxicas de su nombre la raíz tlaxcalli, la cual ha sido traducida al español como tortilla. En las comunidades de dicho lugar existe también una variedad amplísima de la planta del maíz.

El sistema milpa para el cultivo, que paulatinamente ha ido siendo remplazado por métodos de cultivo modernos, es otra de las prácticas compartidas que están ligadas a estas dinámicas de largo tiempo mesoamericanas.

Jauregui, en su crítica ¿Quo vadis, Mesoamérica? (2008), argumenta en contra del concepto ya que plantea que éste fue creado desde una otredad hegemónica con relación a los pueblos originarios en el territorio. Su crítica base es la homogenización teórico-metodológica que se hace de una gran diversidad étnica con el fin político de generar, precisamente, una conceptualización funcional para la integración de múltiples grupos humanos bajo la idea del Estado-Nación Mexicano. El mismo Jauregui intenta rebatir el argumento mesoamericano utilizando el mismo ejemplo del maíz, argumentando que su uso existe en lugares fuera de las fronteras mesoamericanas como el Gran Nayar, tierra de los Coras y los Wirrarika, en el estado de Nayarit México, en la región conocida como Aridoamérica u Oasisamérica.

No es posible negar el uso político que se hizo del término Mesoamérica como fue acuñado por Kirchhoff durante la época de homogenización cultural. Ciertamente fue parte del paradigma de homogenización étnica que resultó en la desaparición de muchas lenguas indígenas. Es el momento de la integración 
1. En lo referente a la vida social, la idea de raza se utilizaba en el siglo XIX como una etiqueta para categorizar a los grupos humanos (y de otras especies animales) según características fisionómicas como el color de piel, la estatura, o el tipo de cabello. Existen muchos ejemplos del uso de este término en las épocas de la homogenización cultural como el poema que Amado Nervo recitó en la cámara de diputados en 1902 titulado precisamente Raza de bronce.

El afán de fomentar la idea de una raza mexicana se relaciona con la necesidad de crear un imaginario social compartido en un territorio con una amplísima diversidad cultural. En la actualidad el concepto raza no es pertinente para las ciencias sociales ya que no existe una vinculación entre las capacidades cognitivas del ser humano con un fenotipo particular. bajo una sola forma cultural, una sola lengua, una sola raza; ${ }^{1}$ el tiempo del paradigma del mestizaje y la modernidad. Pero un mestizaje desde la hegemonía, que borra las formas culturales otras para la reproducción del sujeto humano, consideradas bárbaras o irracionales.

Hoy día se mantiene esa visión utilitaria desde los sujetos-actores hegemónicos, con el mismo objeto discursivo Mesoamérica. Es decir, el uso del concepto para el control del territorio desde los grandes poderes políticos continúa en nuestros días. Evidencia de ello es el llamado Plan Mesoamérica, el cual es una articulación político-económico-militar para la integración regional, pero pensada desde la hegemonía y los paradigmas económicos neoliberales. Paradigmas que conllevan la práctica del despojo de los recursos tanto naturales como humanos. Tal articulación se hace desde los gobiernos de diez países involucrados y fondos económicos transnacionales a través del Banco Interamericano de Desarrollo (Proyecto Mesoamérica, s.f.).

Siendo conscientes del uso político del término y su origen, aceptamos que el objeto discursivo Mesoamérica ciertamente describe la dimensión territorial observada por los sujetos hegemónicos. Sin embargo, sostenemos que la idea mesoamericana, si se enfoca no desde lo utilitario, abarca más que lo observado desde una posición hegemónica. Sostenemos que si la conceptualización sobre el territorio es enfocada desde el paradigma de la reproducción dialógica y recursiva entre sujeto-territorio-cultura, el objeto discursivo Mesoamérica da cuenta de un entramado semiótico-cultural compartido a nivel macro entre los sujetos inmersos en él. Es decir, concebir la reproducción de la cultura, la reproducción de los sujetos y la significación del territorio como procesos ligados entre sí que se influyen y transforman mutuamente hace posible pensar una red de lógicas para entender al mundo vinculadas entre ellas y al mismo tiempo al territorio mesoamericano.

Para observar las lógicas vinculadas a las prácticas de los sujetos, es necesario referirnos a la relación entre cultura y conocimiento. Si consideramos que las prácticas subjetivas están inmersas en lógicas que construyen su sentido, entonces es pertinente pensar que tales sentidos son expresiones de conocimiento que el sujeto posee y que utiliza para la aceptación y comprensión intersubjetivas. Siguiendo esta línea de argumentos, podemos pensar que el conocimiento que los sujetos poseen está relacionado indisolublemente a las lógicas históricas para entender al mundo, que al mismo tiempo son culturales y, por lo tanto, existen en una relación conocimiento-cultura o cognitivo-cultural.

Recuperamos el ejemplo del maíz, no sólo por ser la fuente básica de alimentación en los territorios que forman la región mesoamericana, sino por su profunda simbolización en los procesos cognitivo-culturales de reproducción del sujeto y del territorio. Ciertamente podemos encontrar su uso en el Gran Nayar, o inclusive existen variedades sudamericanas donde se le nombra choclo. Pero es igualmente innegable que la complejidad cognitivo-cultural implica la increíble diversidad del maíz en Mesoamérica deriva de la especialización del conocimiento. En México hay registradas al menos 64 subespecies en datos del 
gobierno mexicano, las cuales en su gran mayoría están relacionadas a la región mesoamericana. En Guatemala, según datos de la prensa, hay 13 subespecies, sin embargo, no existen estudios científico-académicos que hayan mapeado el país completo ni todos los tipos de maíz que se siembran en las distintas épocas del año y climas. Lo anterior sin tomar en cuenta todas las tradiciones de conocimiento gastronómico sobre sus distintas maneras de preparación culinaria, las cuales son altamente especializadas, tanto técnica como simbólicamente.

Por lo tanto, aunque el maíz pueda ser encontrado en otras áreas culturales, la complejidad cognitivo-cultural implicada en su diversidad ciertamente define un área territorial y formas simbólicas particulares. Su existencia en otras áreas es explicable mediante la transmisión de conocimiento inherente al ser humano.

El ejemplo del maíz refiere a uno de los conocimientos heredados del largo tiempo que tejen una relación no solo simbólica, sino político-económica, entre los distintos grupos socioculturales mesoamericanos.

Mesoamérica ha sido siempre un crisol de formas culturales y lingüísticas encarnadas en sujetos concretos que continuamente han estado en contacto entre sí. Esto genera una relación político-social que reproduce formas cognitivas y culturales innegablemente relacionadas que se recrean entre sí, aún en su compleja diversidad. En Guatemala, con su diversidad de 23 lenguas mayas, la mayoría de los considerados indígenas hablan más de una de éstas, ya sea porque sus padres pertenecen a grupos lingüísticos diferentes o por dinámicas económicas. Es difícil encontrar hablantes de sólo una lengua indígena, lo cual es diferente para aquellos que han perdido su lengua y utilizan únicamente el español. Generalmente tal proceso de integración a la forma cultural ladina, o no indígena, implica la pérdida de ciertas dinámicas culturales, pero no de todas ya que hay formaciones sociales de largo tiempo interiorizadas en los sujetos que son mesoamericanas.

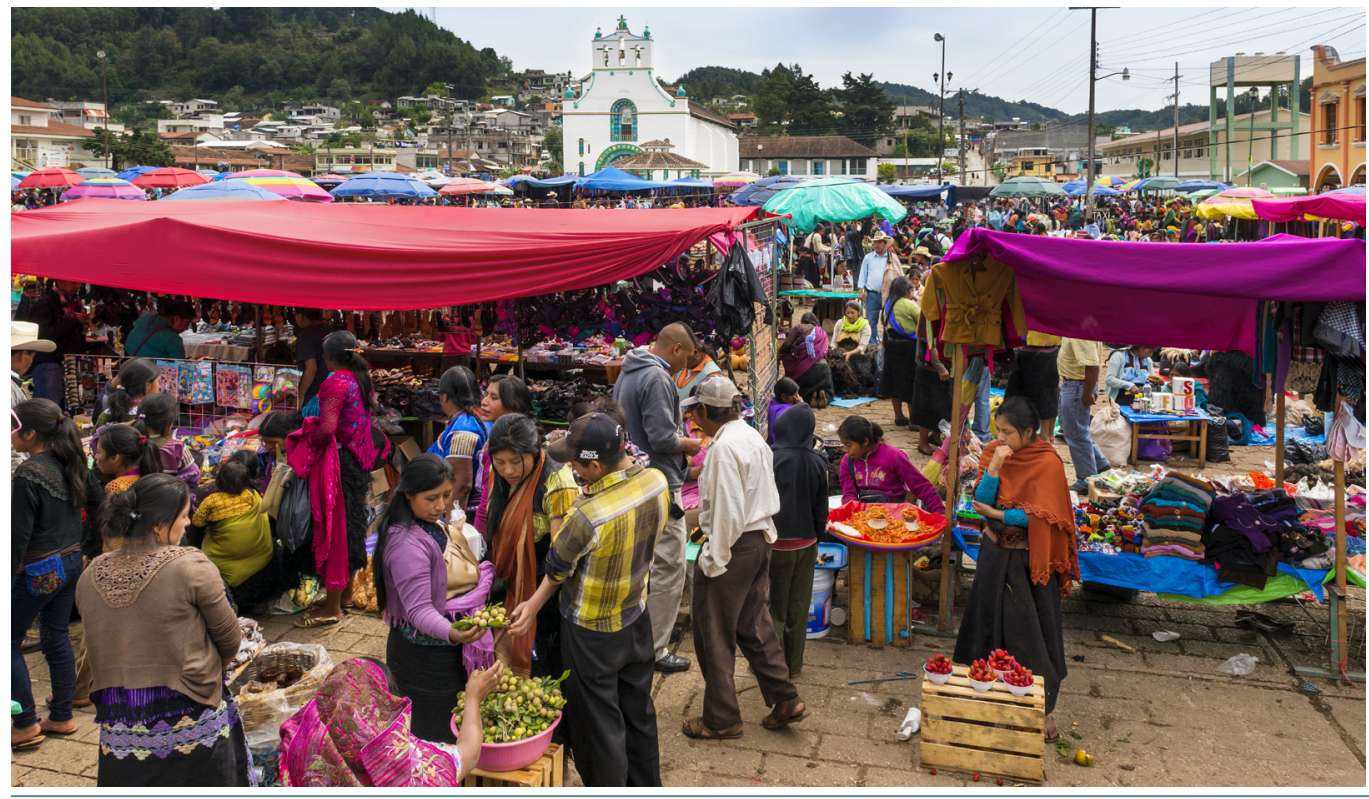


2. El etnocidio es la aniquilación de las lógicas particulares que un grupo posee para entender el mundo. Un ejemplo es la prohibición que existía en España de hablar vasco o catalán durante el gobierno de Francisco Franco (1938-

1973). Tal medida estaba destinada a implantar una sola lógica social y cultural eliminando a las otras visiones e interpretaciones históricas. Es muy común que el etnocidio se relacione con la aniquilación no sólo de las lógicas sociales sino de la población, en cuyo caso es llamado genocidio.
La riqueza de recursos naturales es sólo comparable con la riqueza de la diversidad cultural en Mesoamérica. La articulación de distintas lógicas sociales, históricas, políticas y culturales es algo que ha sido una constante en estos territorios, y a esta dinámica ha de agregarse la constante desigualdad en la historia mesoamericana desde la conquista occidental. Tal proceso de despojo de territorios y etnocidio ${ }^{2}$ ha acompañado desde al menos quinientos años a las dinámicas culturales de reproducción de los sujetos. Dicha coyuntura de guerra ha sido un eje sumamente poderoso en el devenir histórico de la región. Tal magnitud en los eventos conflictivos ha generado pautas simbólicas que se entretejen en la configuración subjetiva, pautas observables en los sujetos hoy día.

Ejemplos de estas lógicas profundas, particulares, han sido mostrados por trabajos etnográficos como El encantamiento de la realidad, donde se postula la existencia de una clave civilizatoria mesoamericana que se expresa transdimensionalmente, a lo largo de las prácticas de la vida cotidiana para los habitantes de los territorios mayas.

Acerca de la clave civilizatoria mesoamericana, observamos que aparece en múltiples aspectos. Primero, en la capacidad de adecuación estratégica para sobrevivir, que el pueblo maya ha desarrollado desde la conquista europea y que se expresa en el continuo: resistencia, adaptación, combinación, innovación y creación. Continuo en el que parecen obrar los mayas en casi todos los aspectos de sus prácticas sociales y que se muestra, por ejemplo, en los modelos de acción política-religiosa frente a la cofradía, en la alcaldía maya, en el sistema de alcaldes auxiliares y posiblemente en algunos esquemas en que operó la patrulla de autodefensa civil (Mendizábal, 2007: 263).

Tal proceso de adaptación estratégica es observable en otros contextos y espacios del territorio mesoamericano como son las mayordomías en los barrios y pueblos de Xochimilco en México, las cuales son remanentes de organizaciones de control de reproducción sociocultural muy parecidas a las cofradías mayas, cada una inmersa en sus propias lógicas particulares.

En el caso de la espiritualidad maya, los llamados sacerdotes mayas o aj $q^{\prime} i j a b$ ', son portadores de conocimientos propios y particulares de la región mesoamericana, esto se materializa en la realización de rituales y ceremonias que condensan lógicas sociales, políticas y religiosas, que se llevan a cabo en la actualidad, son rastreables en el largo tiempo hasta épocas prehispánicas (Mendizábal, 2015). Estos contextos rituales y ceremoniales se relacionan con la cuenta calendárica mesoamericana de 260 días, lo cual evidencia la permanencia de estos conocimientos ancestrales en las comunidades contemporáneas.

Aj q 'ijab' es un término maya que refiere a una colectividad de sujetos que realizan una diversidad de prácticas rituales y ceremoniales articuladas en prácticas particulares como es la cuenta calendárica mesoamericana, el uso ceremonial del fuego, la danza ritual y las ofrendas a los ajaw o señores de los días, entre otras. Las consultas que se hacen a los ajaw son peticiones de respuesta a 
preguntas concretas de sujetos pertenecientes al grupo sociocultural que cree y funciona en la lógica transcultural del aj q'ijab'. Un ejemplo podría ser las peticiones de consulta sobre si es factible o no el viaje hacia Estados Unidos de un muchacho maya, o la consulta sobre si un matrimonio tendrá o no un futuro. Tales ejemplos dan cuenta de la continuidad entre lo que desde la lógica occidental es concreto y observable, como podría ser un riesgoso viaje o un matrimonio, con las lógicas ancestrales enraizadas al largo tiempo, articuladas en una dimensión de producción de sentido sociocultural que a lo largo de este artículo hemos denominado mesoamericano.

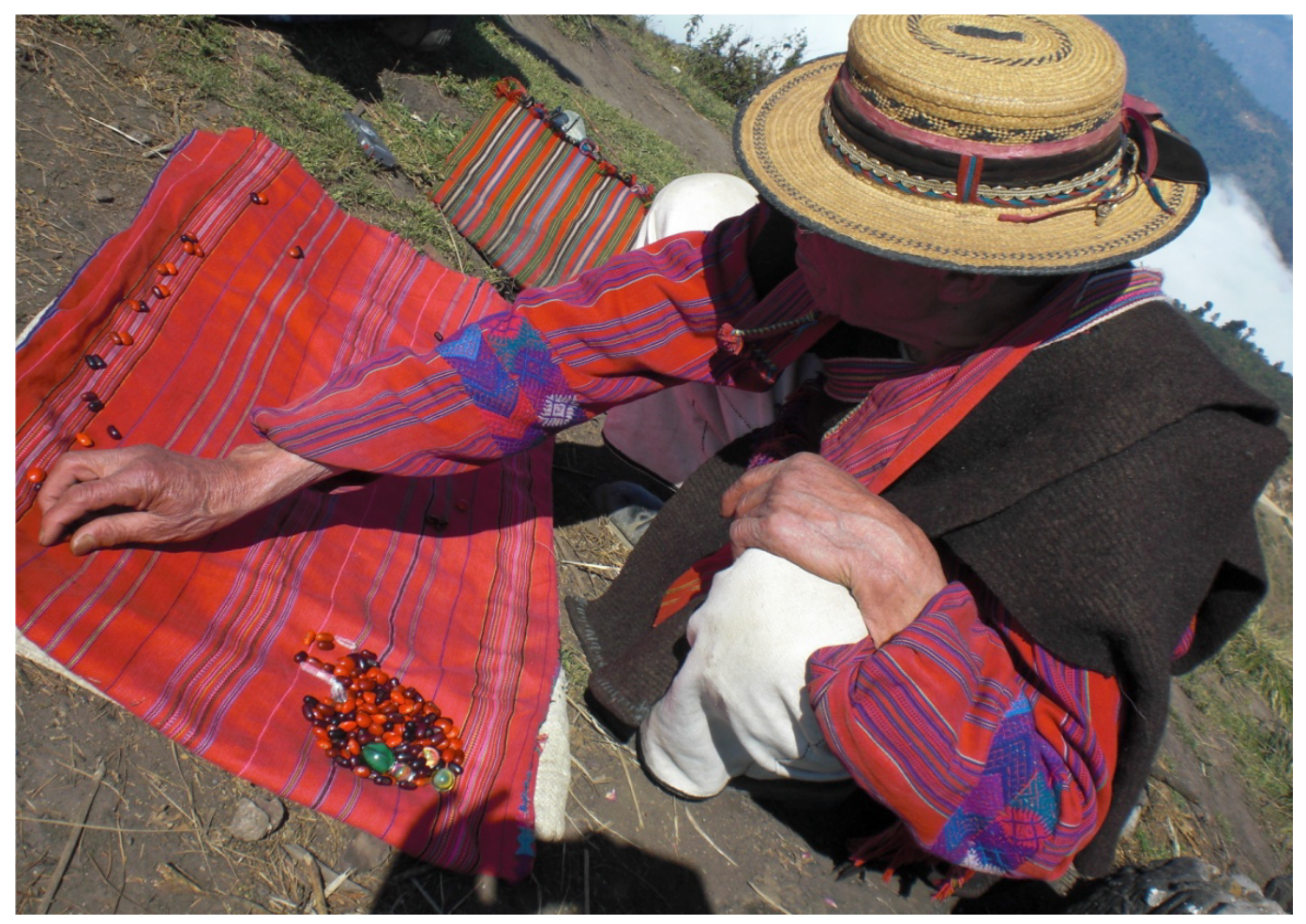

Las lógicas mesoamericanas no suceden solamente en una direccionalidad temporal hacia el pasado; al estar relacionadas con prácticas contemporáneas, estas dinámicas de producción de sentido generan una prospección hacia el futuro. La multidimensionalidad de las características que en el presente artículo llamamos mesoamericanas hace observable una dinámica semiótica que refiere a distintos estratos de temporalidad cultural. Momentos de generación de sentido que se apoyan en conocimientos ancestrales de las culturas observadas con relación a sus territorios e historia. Así mismo son prácticas contemporáneas con una relación directa a las coyunturas sociopolíticas en las cuales suceden. Y, por lo anterior, plantean, aun de manera no consciente, escenarios futuros para su reproducción.

La relación entre el pasado, el presente y el futuro y la transdimensionalidad de estas prácticas generadoras de sentido son equiparables a las características que Lotman plantea sobre los procesos simbólicos: 
[...] al atravesar el espesor de las culturas, el símbolo se realiza en su esencia invariante. En este aspecto podemos observar su repetición. El símbolo actuará como algo que no guarda homogeneidad con el espacio textual que lo rodea, como un mensajero de otras épocas culturales (=otras culturas), como un recordatorio de los fundamentos antiguos (= "eternos") de la cultura. Por otra parte, el símbolo se correlaciona activamente con el contexto cultural, se transforma bajo su influencia y, a su vez, lo transforma. Su esencia invariante se realiza en las variantes (Lotman, 1996 :146).

Así, podemos plantear la dimensión simbólica de las características mesoamericanas realizadas en la práctica de los sujetos. Es decir, son elementos que refieren a dimensiones profundas y ligadas al largo tiempo, pero que se realizan de una manera variable, con relación al cuándo, cómo y dónde suceden.

Como pudo apreciarse a lo largo del presente artículo, el pensamiento complejo y la transdisciplinariedad son sumamente útiles al momento de acercarnos a los procesos de reproducción de la cultura. Procesos complejos que permiten pensar conceptos transdimensionales como Mesoamérica, así como la existencia de sujetos complejos. En síntesis, Mesoamérica implica la homogeneidad y la heterogeneidad, en un continuum que la define.

\section{Referencias}

- Duverger, C. (2007). El primer mestizaje. La clave para entender el pasado mesoamericano, México, CONACULTA/INAH/UNAM/Taurus.

- Haidar, J. (2006). Debate CEU-Rectoría, torbellino pasional de los argumentos, México, UNAM.

* Jauregui, J. (2008). ¿Quo vadis Mesoamérica? Recuperado de https://revistas.inah. gob.mx/index.php/antropologia/article/view/2892/2793, Secretaría de Cultura/ INAH, México.

* Kirchhoff, P. (2000). Mesoamérica. Dimensión Antropológica. Vol. 19, mayo-agosto, pp. 15-32. Recuperado de http://www.dimensionantropologica.inah.gob. $\mathrm{mx} / \mathrm{p}=1031$

* Kirchhoff, p. (1943). Mesoamérica, México, ENAH/Aguirre y Beltrán editores.

* Lotman, I. M. (1996). La semiosfera I [edición y traducción de Desiderio Navarro]. Madrid: Ediciones Cátedra.

* Mendizábal, H. (2015). ¿Cómo se habla con los dioses? Análisis de una ceremonia maya en el altiplano guatemalteco. Una aproximación de la etnosemántica hacia la semiótica de la cultura. Tesis de licenciatura. México: ENAH.

* Mendizábal, S. (2007). El encantamiento de la realidad. Guatemala: Programa de Educación Intercultural Multilingüe de Centroamérica/Universidad Rafael Landívar. 
* Mendizábal, S. (2010). El Kaba’wil en nuestra historia. Guatemala, Universidad Rafael Landívar, Instituto de Estudios Humanísticos.

* Morin, E. (1999). El método III el conocimiento del conocimiento, Madrid: Ediciones Cátedra

- Palencia, S. (2014). Mesoamérica: concepto y lucha aproximación histórico-social desde la teoría crítica, Del sujeto subalterno al sujeto cosmológico: un espejo para transformarnos. Guatemala: Universidad Rafael Landivar, Instituto de Estudios Humanísticos.

- Proyecto Mesoamérica (sin fecha). ¿Cómo se financian los proyectos? Portal oficial proyecto Mesoamérica. El Salvador. Recuperado de http://proyectomesoamerica. org/joomlal.

* Zamora, M. (2014). La relación entre cultura-naturaleza en la transformación de una sociedad orientada hacia un buen vivir: un estado de la cuestión desde la Mesoamérica contemporánea, Del sujeto subalterno al sujeto cosmológico: un espejo para transformarnos. Guatemala: Universidad Rafael Landívar, Instituto de Estudios Humanísticos.

\section{Cómo citar este artículo}

* Mendizábal García, H. E. (2018). Los procesos de reproducción cultural en Mesoamérica, una perspectiva transdisciplinaria. Revista Digital Universitaria (RDU). Vol. 19, núm. 2 marzo-abril. DOI: http://doi.org/10.22201/codeic.16076079e.2018. v19n2.a1. 